\title{
Article
}

\section{Fizzle Testing: An Equation Utilizing Random Surveillance to Help Reduce COVID-19 Risks}

\author{
Christopher A. Cullenbine ${ }^{1}$, Joseph W. Rohrer ${ }^{2}$, Erin A. Almand ${ }^{2, *} \mathbb{}$, J. Jordan Steel ${ }^{2}$, Matthew T. Davis ${ }^{1}$, \\ Christopher M. Carson ${ }^{1}$, Steven C. M. Hasstedt ${ }^{2}$, John C. Sitko ${ }^{2}$ and Douglas P. Wickert ${ }^{3}$ \\ 1 Department of Mathematical Sciences, United States Air Force Academy, Colorado Springs, CO 80840, USA \\ christopher.cullenbine@usafa.edu (C.A.C.); Matthew.Davis@usafa.edu (M.T.D.); \\ christopher.carson@usafa.edu (C.M.C.) \\ 2 Department of Biology, United Stated Air Force Academy, Colorado Springs, CO 80840, USA; \\ Joseph.rohrer@usafa.edu (J.W.R.); james.steel@usafa.edu (J.J.S.); steven.hasstedt@usafa.edu (S.C.M.H.); \\ john.sitko@usafa.edu (J.C.S.) \\ 3 Department of Aeronautical Engineering, United States Air Force Academy, \\ Colorado Springs, CO 80840, USA; Douglas.wickert@usafa.edu \\ * Correspondence: Erin.Almand@usafa.edu; Tel.: +1-719-333-6029
}

Citation: Cullenbine, C.A.; Rohrer, J.W.; Almand, E.A.; Steel, J.J.; Davis, M.T.; Carson, C.M.; Hasstedt, S.C.M.; Sitko, J.C.; Wickert, D.P. Fizzle Testing: An Equation Utilizing Random Surveillance to Help Reduce COVID-19 Risks. Math. Comput. Appl. 2021, 26, 16. https://doi.org/10.3390/ mca26010016

Received: 9 December 2020

Accepted: 9 February 2021

Published: 13 February 2021

Publisher's Note: MDPI stays neutral with regard to jurisdictional claims in published maps and institutional affiliations.

Copyright: (c) 2021 by the authors. Licensee MDPI, Basel, Switzerland. This article is an open access article distributed under the terms and conditions of the Creative Commons Attribution (CC BY) license (https:// creativecommons.org/licenses/by/ $4.0 /)$.

\begin{abstract}
A closed-form equation, the Fizzle Equation, was derived from a mathematical model predicting Severe Acute Respiratory Virus-2 dynamics, optimized for a 4000-student university cohort. This equation sought to determine the frequency and percentage of random surveillance testing required to prevent an outbreak, enabling an institution to develop scientifically sound public health policies to bring the effective reproduction number of the virus below one, halting virus progression. Model permutations evaluated the potential spread of the virus based on the level of random surveillance testing, increased viral infectivity and implementing additional safety measures. The model outcomes included: required level of surveillance testing, the number of infected individuals, and the number of quarantined individuals. Using the derived equations, this study illustrates expected infection load and how testing policy can prevent outbreaks in an institution. Furthermore, this process is iterative, making it possible to develop responsive policies scaling the amount of surveillance testing based on prior testing results, further conserving resources.
\end{abstract}

Keywords: COVID-19; SEIR; simulation; surveillance

\section{Introduction}

As Severe Acute Respiratory Virus-2 (SARS-CoV-2), the causative agent behind Coronavirus Disease-19 (COVID-19), continues to disrupt life globally, scientists are beginning to understand principles underlying the movement of the virus through populations, and plan accordingly. As the understanding of this virus grows, universities, businesses and society at large are turning to science to inform their ability to return to everyday life safely. The basic reproduction number $\left(R_{0}\right)$ of SARS-CoV-2 is highly variable and governed by numerous biological, behavioral and environmental factors [1-3]. When evaluating infectious diseases over time, it is important to move past a static concept of $R_{0}$ that only applies to a snapshot in place and time, and evaluate the time-dependent variation, or effective reproduction number $\left(R_{e f f}\right)$. Average estimates hover around 2.2 [4], but climb to temporal values as high as 10 in congested areas with minimal mitigation strategies $[5,6]$. This number takes into account a decline in susceptible individuals as time progresses (intrinsic factors) in addition to targeted control measures to prevent the spread of disease (extrinsic factors). Infectious diseases continue to spread through populations unless the $R_{\text {eff }}$ is below one [7].

By identifying extrinsic factors recommended by Public Health (e.g., social distancing, masks, enhanced cleaning policies, remote working) and combining them with a targeted 
quarantine and isolation strategy (limiting exposure to susceptible individuals), it is possible to drive $R_{\text {eff }}$ below 1, "fizzling" out an outbreak, as the rate of new cases is less than the rate of recovery. Despite the efficacy of these mitigation strategies, a growing body of evidence suggests a significant asymptomatic or pre-symptomatic transmission rate of SARS-CoV-2, especially amongst a younger population [8,9]. To combat this population covertly spreading the virus, a level of surveillance testing is necessary to determine the prevalence in a community and remove asymptomatic carriers from the susceptible population.

This study identified variables likely to affect viral transmission, and overlaid them on a social network common to the university setting. We developed, verified, and validated a stochastic susceptible (S), exposed (E), infected (I) and recovered (R) model developed by the University of Washington, adjusted for our population [10]. Finally, we demonstrated an empirical method to determine the minimum level of surveillance testing required to fizzle viral spread and used the SEIR model to validate our methodology.

\section{Materials and Methods}

Assumptions based on current literature, direct observation, and historical precedents surrounding SARS-CoV-2 and other coronaviruses [11,12], were refined for our population of 4000 students (low risk, medically screened 18-26 years old) and 1000 faculty or staff members, as seen in Table 1. Some of the parameters, e.g., "Days b/w test", are used solely to explore the impact of different surveillance test planning options (e.g., testing every day vs. once a week). With these variables, a stochastic SEIR compartmental model (Appendix A) based on social network representations of the US Air Force Academy was used to evaluate mitigation and control policy options for the institution [10]. This model was run through the minimum and maximum value of each parameter, to elucidate the overarching effect of each parameter on the results. A Monte Carlo simulation of daily activities that included interactions between agents capable of transmitting a disease gave similar results to the SEIR compartmental model.

Table 1. Parameters for Model Comparisons and Validation. The best estimate column provides the values chosen for modeling the described population.

\begin{tabular}{ccccc}
\hline Parameter & Description & Best Estimate & Range Modeled & Comments \\
\hline$d_{0}$ & Start Date & $12 \mathrm{Aug}$ & - & Day zero of simulation runs \\
$R_{0}$ & Initial $R$ & 1.6 & $1.2-3.5$ & Initial basic reproduction number \\
$N$ & Population & 5000 & $2000-5000$ & Population size (students, faculty, and staff) \\
$I_{0}$ & Initial Infected & 0 & $5-10$ & Initial infected (Asymptomatic) \\
$\alpha_{\mathrm{a}}$ & Asymptomatic & $50 \%$ & $20-100 \%$ & Percentage of infected individuals \\
$\phi$ & remaining asymptomatic \\
$\omega_{\mathrm{w}}$ & Contact Tracing & $10 \%$ & $10-50 \%$ & Close contact tracing effectiveness \\
$\delta$ & New I's & 1 & $0.25-5$ & Exogenous weekly infection rate $\left(-w \mathrm{w}^{-1}\right)$ \\
$p$ & Days b/w test & 1 & $1-14$ & Days between baseline testing (pooled) \\
$1-p$ & Mixing & 0.3 & $0.2-0.8$ & Likelihood of infection from random interaction \\
& Mixing & 0.7 & $0.2-0.8$ & Likelihood of infection from close contact \\
$q$ & Mixing & 0.3 & $0.2-0.8$ & Likelihood of infection from random interaction \\
& & & with quarantined or isolated individuals (Q\&I) \\
\hline
\end{tabular}

We verified the model by comparing outputs with the available data from the outbreaks on the USS Theodore Roosevelt (Appendix B, Figure A2), the French aircraft carrier Charles de Gaulle, and the Diamond Princess cruise ship [5,11,13]. The aircraft carrier outbreaks occurred in isolated, closed populations of the same size as our population ( 5000 individuals) with similar demographics (predominately young sailors, our students; and some older sailors, our faculty and staff). Parameters relating to biological transmission and social behavior were fitted to the available public data. The modeled social network structure of the US Air Force Academy was validated by comparing simulations of a norovirus outbreak with the actual outbreak that occurred in November 2019. Norovirus 
has well-defined transmission dynamics (incubation time, infectious period, $R_{0}$ ) so, it was possible to isolate and confirm the underlying social network and behavioral factors of our population (Appendix B, Figure A2). The final values of parameters used for establishing SARS-CoV-2 mitigation and policy measures are given in Table 1 and Appendix A. The USS Theodore Roosevelt outbreak and stochastic simulation comparison is given in Appendix B, Figure A2; however, the derivation of the Fizzle Equation, detailed below, is not dependent on the computer model. The model merely provided a convenient means to demonstrate that surveillance testing at the fizzle condition is sufficient to prevent a nascent outbreak from developing and testing below the fizzle condition is insufficient to prevent exponential spread.

IRB determination on the surveillance testing and mathematical modeling (FAC20200024N and FAC20200025N, respectively) deemed this study as Not Humans Subject Research in accordance with 32 CR 219, DoDI3216.02 and AFI 40-402.

\section{Results}

Modifying the SEIR compartmental model to include an isolation compartment for (1) infections detected and identified through surveillance testing, (2) symptomatic individuals, and (3) infections identified during testing of contact traced individuals, offers a strategy for establishing a baseline surveillance test rate to fizzle any nascent outbreak. The goal of the fizzle strategy is to reduce the number of infected circulating in the population and reduce the effective reproductive number, $R_{\text {eff, }}$, to less than one. The Fizzle Equation then permits policy makers to establish a minimum level of surveillance testing to avoid an outbreak based on expected virus parameters and other population characteristics (e.g., symptomatic/asymptomatic rates and contact tracing effectiveness). The derivation of the Fizzle Equation is as follows.

Including an isolation compartment, $D$, for detected infections modifies the usual SEIR equations:

$$
\begin{gathered}
\frac{d S}{d t}=-\beta I \frac{S}{N} \\
\frac{d E}{d t}=\beta I \frac{S}{N}-\sigma E-\Theta_{E} \Psi_{E} E \\
\frac{d I}{d t}=\sigma E-\gamma I-\Theta_{I} \Psi_{I} I \\
\frac{d R}{d t}=\gamma I+\gamma D_{I} \\
\frac{d D_{E}}{d t}=\Theta_{E} \Psi_{E} E-\sigma D_{E} \\
\frac{d D_{I}}{d t}=\Theta_{I} \Psi_{I} I+\sigma D_{E}-\gamma D_{I}
\end{gathered}
$$

where $S, E, I$, and $R$ are the number of susceptible, exposed, infectious, and recovered individuals in a population of $N$ people. $D_{E}$ and $D_{I}$ are the detected infections from the exposed and infectious compartments, respectively, $\beta$ is the transmission rate, $\sigma$ is the latent rate at which the exposed become infected (after an incubation period), $\gamma$ is the rate at which the infected recover from the disease, $\Psi$ is test effectiveness (combines test sensitivity and collection efficacy) and $\Theta$ is the equivalent test rate that combines random surveillance testing, symptomatic test rate, and contact tracing test rate. Specifically,

$$
\Theta_{I}=\Theta_{e q}=\theta+\alpha_{s} \theta_{s}+\phi \eta \theta+\phi \eta \alpha_{s} \theta_{s}
$$

where $\theta$ is the baseline (random) surveillance test rate, $\theta_{S}$ is the rate that symptomatics volunteer for testing, $\alpha_{s}$ is the proportion of the population that experiences symptoms, $\phi$ is the contact tracing effectiveness and $\eta$ is an efficiency parameter that accounts for close contact redundancy between different infected individuals. For sufficiently large 
populations, and a low infection prevalence, $\eta$ will be close to unity. As an epidemic becomes more widespread and the network graph of infectious close contacts overlaps with other infected nodes, $\eta$ will decrease to some value less than 1 . In all cases, the number of infected identified through contact tracing cannot exceed the total number of individuals infected. The $\eta$ efficiency parameter ensures this is the case.

The effective reproductive number, $R_{\text {eff, }}$ can be estimated by the spectral radius of the next-generation operator [14]. The rate of the change of $x_{i}^{\text {th }}$ compartment can be written as $F_{i}-V_{i}$ where $F_{i}$ is the rate of appearance of new infections in the $i$ th compartment and $V_{i}$ is the transfers between compartments. The next-generation matrix is then $G=F V^{-1}$ where $F=\left[\partial F_{i} / \partial x_{j}\right]$ and $V=\left[\partial V_{i} / \partial x_{j}\right]$. Considering only the two infected compartments, $E$ and $I$, the appearance and transfer matrices are

$$
\begin{gathered}
F=\left[\begin{array}{ll}
0 & \beta \\
0 & 0
\end{array}\right] \\
V=\left[\begin{array}{cc}
\sigma+\Theta_{E} \Psi_{E} & 0 \\
-\sigma & \gamma+\Theta_{I} \Psi_{I}
\end{array}\right]
\end{gathered}
$$

With testing, the effective reproductive number, $R_{\text {eff, }}$ is the spectral radius (dominant eigenvalue).

$$
R_{e f f}=\rho\left(F V^{-1}\right)=\frac{\beta \sigma}{\left(\gamma+\Theta_{I} \Psi_{I}\right)\left(\sigma+\Theta_{E} \Psi_{E}\right)}
$$

For a nascent outbreak to fizzle, $R_{\text {eff }}<1$. In establishing the required surveillance test rate for an outbreak of a novel virus to fizzle, the population is taken as fully-susceptible. For surveillance of SARS-CoV-2, the additional contribution from possible detection of exposed individuals from random testing can be reasonably neglected. Noting that in the absence of testing the basic reproductive number, $R_{0}=\frac{\beta}{\gamma}$ as expected, (10) may be solved to yield the Fizzle Equation:

$$
\Theta_{e q}=\left(\theta+\alpha_{a} \theta_{s}\right)(1+\phi \eta) \geq \frac{1}{\psi} \gamma\left(R_{0}-1\right)
$$

where $\Theta_{e q}$ is the equivalent testing (7) that combines all types of testing: asymptomatic surveillance testing $\theta$, symptomatic diagnostic testing $\theta_{s}$, and contract tracing $\phi$.

The Fizzle Equation was evaluated using the SEIR model (term definitions in Table 2) for thousands of permutations across a range of parameters (Table 1). Each run was seeded with different random numbers generating different outbreak responses. In all cases, nascent outbreaks fizzled for all runs in which the baseline testing met or exceeded the fizzle condition given by (11). Figure 1 depicts the baseline daily test rate for asymptomatic individuals as a function of basic reproductive number (assuming a totally susceptible population) and contact tracing effectiveness for a range of asymptomatic rates.

Table 2. Susceptible, Exposed, Infected, and Recovered (SEIR) Model Parameters and Values used in Figure 1. The baseline test rate range was chosen based on requirement to fizzle an outbreak.

\begin{tabular}{ccc}
\hline Parameter & Description & Range Modeled \\
\hline$\theta$ & Baseline test rate & $3-21 \%$ \\
$\phi$ & Contact tracing effectiveness & $0-50 \%[15]$ \\
$\alpha_{a}$ & Infectious asymptomatic rate & $20-90 \%[16]$ \\
$\psi$ & Overall test effectiveness rate (sensitivity and collection) & $85 \%[17]$ \\
$\gamma^{-1}$ & Duration of infectiousness & $10[18]$ \\
$R_{0}$ & Basic reproduction number & $3.0[19]$ \\
\hline
\end{tabular}




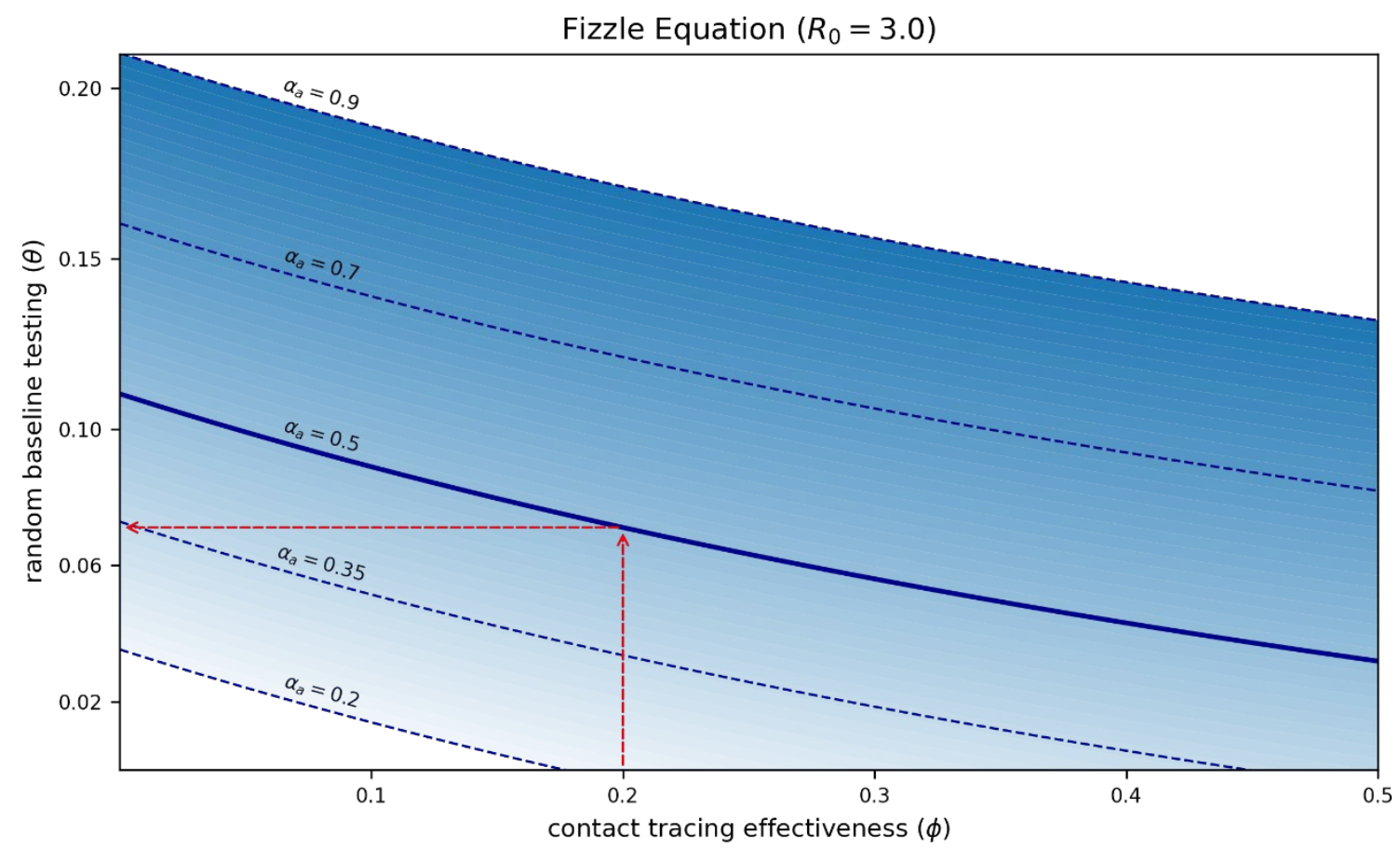

Figure 1. Solution of the Fizzle Equation for values in Table 2. The random baseline testing $(\theta)$ is the daily test rate contact tracing effectiveness for a range of asymptomatic rates for a basic reproductive number of 3.0. For a given contact tracing effectiveness and asymptomatic rate, testing at or above the indicated baseline test rate will ensure an effective reproductive number of less than unity and that any nascent outbreak fizzles rather than grows exponentially. For this instance, when the reproductive number is 3.0 , with a $20 \%$ efficiency at contact tracing effectiveness, and $50 \%$ of the population is asymptomatic, the daily baseline testing rate is $7 \%$, as shown by the red arrows.

\section{Discussion}

The variability of the virus and behavioral factors in diverse institutions make predictions on the amount of testing required difficult. However, as demonstrated here, with careful validation it is possible to model the dynamics of SARS-CoV-2, allowing community leaders to predict the spread of SARS-CoV-2 through different populations, and make informed policy decisions based on scientific evidence. Specifically, our Fizzle Equation provides a direct way to determine the amount and type of testing in a population required to prevent outbreaks.

Our results illustrate that surveillance testing on asymptomatic individuals is crucial to prevent the spread of SARS-CoV-2 and creates a greater impact on containing outbreaks rather than contact tracing. Modeling this relationship creates a more effective allocation of resources at institutions with similar dynamics to our population, i.e., universities, cruise ships and otherwise isolated communities.

As seen in Figure 1, contact tracing effectiveness is a fundamental variable in determining the amount of random testing required. In a scenario with numerous personnel able to dedicate time and resources to contact tracing, it is a valuable alternative to random testing. However, limitations in determining efficacy of contact tracing have led to conservative estimates for contract tracing effectiveness. Preliminary efficacy data from the implementation of contact tracing at our university supports our initial assumptions (approximately $60 \%$ of SARS-CoV-2 infections were identified on the basis of random surveillance testing, $25 \%$ from contact tracing, and $15 \%$ of cases from symptomatic individuals). For example, without random surveillance testing, at a 35\% asymptomatic rate, a low estimate for SARSCoV-2 [20], the contact tracers would need to identify $45 \%$ of the individuals coming into contact with each infected person. If that rate jumps to a $50 \%$ asymptomatic population, a more likely scenario, contact tracing needs to identify well over half of the individuals an 
infected individual came into contact with. In this instance, contact tracing is an adjunct, but not sufficient by itself for outbreak control.

The complimentary option in our scenario, with a $90 \%$ asymptomatic population and an initial $R_{0}$ of 3.0, is surveillance testing, and when used alone this requires testing $20 \%$ of the population daily - a daunting and expensive option for most institutions. Fortunately, surveillance testing is used in conjunction with, but not as a replacement for, symptomatic screening and contact tracing. Our Fizzle Equation demonstrates that this combination reduces the daily surveillance testing burden to $7 \%$ of the population, a much more manageable workload. Surveillance testing represents a particularly powerful tool for pathogens with a high proportion of asymptomatic carriers, such as SARS-CoV-2, because each individual pulled out of circulation due to random surveillance is an individual that otherwise would have remained infectious without being isolated.

The advantages of surveillance testing are so robust that even when expected test sensitivity was decreased in our model, it was still possible to fizzle any outbreaks. This finding implies that pooled testing, which typically reduces sensitivity, is a viable strategy for increasing the efficiency of a surveillance testing program. A pooled testing strategy informed by Hitt et al. [19] was developed based on predicted individual test sensitivity, specificity, and expected disease prevalence within the population. While this approach loses the ability to initially say which individual sample is positive, the low viral prevalence (expected to be $\sim 0.5-1 \%$ ) means the majority of samples and therefore pools will yield negative test results. When a positive pool is identified, the individual samples can then be retested separately to identify the infected individual(s). Thus, the minimum number of tests required to surveil a population using this approach is a function of pool size and prevalence. As prevalence grows, pool size must decrease. Likewise, as prevalence decreases, pool size should increase in order to maintain the optimal testing configuration and consume less test material over the long term.

Furthermore, an active surveillance testing program provides regular updates to the estimated prevalence of infections in a population which may be used to drive policy decisions in a continuous feedback loop. Institutions may vary the surveillance and contact tracing testing rates to conserve resources as infection load increases or decreases in the population. Knowing the prevalence in the community also enables decision-makers to determine the efficacy of the control measures in place and strike a balance between public health and safety on one hand, and mental health and morale on the other.

This model also helps leaders allocate limited infrastructure for quarantine and isolation, by providing predictions on the number of exposed and known infected individuals within a given confidence interval. Decision makers may anticipate and reserve adequate space to quarantine and isolate infectious individuals to stem the virus spread. This information, in addition to the amount of time before the peak infection, may be seen in Figure 2, which annotates the values and information typically gleaned from a simulation run. To demonstrate the effectiveness of testing at the fizzle condition, Figure 3 compares transmission dynamics of SARS-CoV-2 through a 5000-person population with surveillance testing at and below the fizzle condition.

While morbidity and mortality are of great concern for SARS-CoV-2, and subsequently COVID-19, a limitation of this study is not knowing the severity of disease in the infected individuals. This equation and modeling approach does not directly take into account different populations, as it is targeting a healthy, low risk, medically-screened group of 18-26-year-olds. If this equation and approach is utilized in a higher-risk setting, public health data for the relevant age ranges should be taken into account, as the risk to an individual may be higher even with few cases. Additionally, the planning parameters for implementation here focused on the near-term, so reinfection rates were kept at zero. As additional scientific evidence emerges on the topic of reinfection, this parameter may play a larger role [21,22]. 


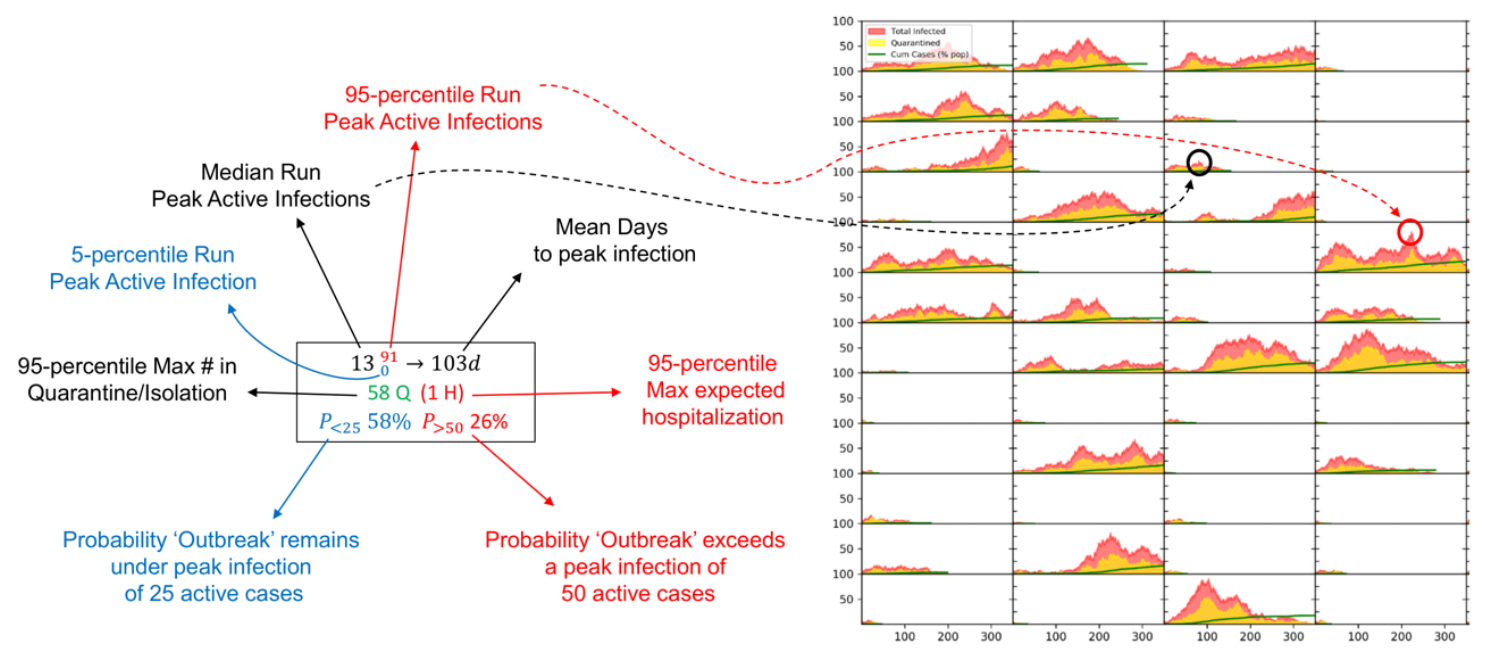

Figure 2. Table of key values highlighting variation in the modeling runs and typical statistics to inform policy decisions. \#Q denotes the individuals in quarantine or isolation and the \#H shows the number of infected individuals requiring hospitalization. Other variables show variation in the modeling outcomes, as described. For this series of runs, the $R_{0}=2.2$, $I_{0}=5, \theta=5 \%$, and $\phi=20 \%$. On the graphs, red depicts total number of infected, yellow is total number of individuals in quarantine and isolation, and the green line is cumulative cases as a percentage of the total population.

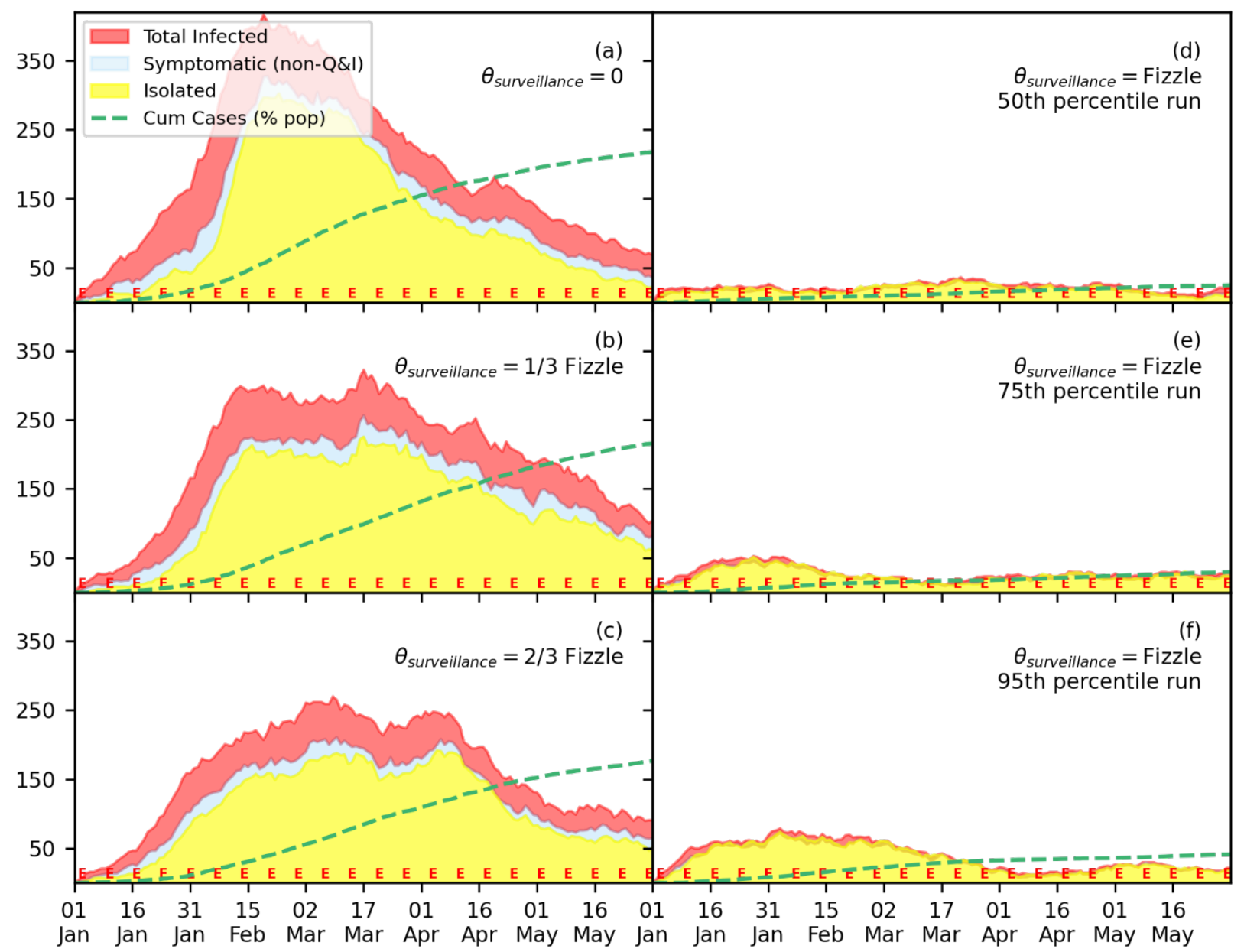

Figure 3. Demonstration of surveillance testing at and below the Fizzle condition. $R_{0}=3.0,50 \%$ asymptomatic rate, $20 \%$ contact tracing effectiveness, and $85 \%$ test effectiveness for the infected compartment. Results are the median run (ordered by peak infection) of 100 simulations of the stochastic SEIR model except as noted. Cumulative cases are depicted as percentage 
of the entire population (top of $\mathrm{y}$-axis is 100\%). Population size is 5000; initial conditions include 25 exposed on 1 Jan (0.5\% of population) and 2 new random exposed cases introduced every week (depicted by red E). (a) No surveillance testing (symptomatic individuals are identified through volunteering of symptoms and are contact traced with the same contact tracing effectiveness); (b) daily surveillance testing at $1 / 3$ the predicted fizzle rate; (c) daily surveillance testing at $2 / 3$ the predicted fizzle rate; (d) testing at the fizzle rate (median run); (e) testing at the fizzle rate (75th-percentile run); (f) testing at the fizzle rate (95th-percentile run).

\section{Conclusions}

With the high asymptomatic rate of individuals infected with SARS-CoV-2, surveillance testing is required to identify individuals that do not appear sick, but are still capable of spreading the virus. Our derived Fizzle Equation lays bare the level of testing required to prevent an outbreak. Mathematical modeling tailored to specific populations confirmed that employing testing in the manner prescribed by the Fizzle Equation is effective at preventing outbreaks while preserving resources. Furthermore, this approach takes into account potential reduced test sensitivity, either from collection type, detection methodology or pooled testing, providing a scaled approach for a variety of applications.

Author Contributions: Conceptualization, C.A.C., M.T.D., C.M.C., E.A.A., J.C.S., and D.P.W.; methodology, J.W.R., C.A.C., E.A.A., J.J.S., M.T.D., C.M.C., J.C.S. and D.P.W.; software, C.A.C., M.T.D., C.M.C., J.C.S. and D.P.W.; validation, C.A.C., M.T.D., C.M.C. and D.P.W.; formal analysis, C.A.C. and D.P.W.; investigation, C.A.C., M.T.D., C.M.C., J.C.S. and D.P.W.; resources, E.A.A., J.J.S., J.C.S. and S.C.M.H.; data curation, C.A.C., M.T.D., C.M.C., J.C.S. and D.P.W.; writing-original draft preparation, J.W.R.; writing-review and editing, E.A.A.; visualization, C.A.C. and D.P.W.; supervision, S.C.M.H. and D.P.W.; project administration, S.C.M.H. and D.P.W.; funding acquisition, E.A.A., J.J.S., S.C.M.H. and J.C.S. All authors have read and agreed to the published version of the manuscript.

Funding: This research received no external funding.

Acknowledgments: The 10th Medical Group, collected the samples and managed the quarantine and isolation plans. Additionally, members of the Pandemic Math Team provided inputs and model refinements. The views expressed in this article are those of the author and do not reflect the official policy or position of the United States Air Force, Department of Defense, or the U.S. Government.

Conflicts of Interest: The authors declare no conflict of interest.

\section{Appendix A}

Table A1. SEIR-Model Parameters for Simulation of SARS-CoV-2 Dynamics at the USAF Academy. For a full discussion of the parameters and the model see [10].

\begin{tabular}{|c|c|c|}
\hline Parameter & Description & $\begin{array}{c}\text { Initial Parameter Value or } \\
\text { Modeled Range }\end{array}$ \\
\hline$S$ & Number of susceptible individuals & $N-E$ \\
\hline$E$ & Number of exposed individuals & $1-5 \%$ of $N$ \\
\hline$I$ & Number of infectious individuals & 0 \\
\hline$D_{E}$ & Number of exposed individuals with detected cases & 0 \\
\hline$D_{I}$ & Number of infected individuals with detected cases & 0 \\
\hline$R$ & Number of recovered individuals & 0 \\
\hline$F$ & Number of infection-related fatalities & 0 \\
\hline$N$ & Total number of living individuals & 5000 \\
\hline$\beta$ & Rate of transmission & $0.12-0.16$ \\
\hline$\beta_{\text {asymp }}$ & Rate of asymptomatic transmission & $20-80 \% \beta_{\text {sym }}$ \\
\hline$\sigma^{-1}$ & Incubation period (upon exposure) & $2-5$ days \\
\hline$\gamma^{-1}$ & Duration (reciprocal of recovery period) & 5-14 days \\
\hline$\mu$ & Rate of infection-related deaths & 0 \\
\hline$\theta_{E}$ & Rate of baseline testing (for exposed individuals) & varies \\
\hline$\theta_{I}$ & Rate of baseline testing (for infectious individuals) & varies \\
\hline$\phi$ & contact tracing effectiveness (probability of finding a positive individual from list of close contacts) & $5-50 \%$ \\
\hline$\psi_{I}$ & Infected compartment test effectiveness (combines test sensitivity and sample efficacy)) & $80-95 \%$ \\
\hline$\psi_{E}$ & Exposed compartment test effectiveness (combines test sensitivity and sample efficacy)) & $0-15 \%$ \\
\hline$q$ & Probability of isolated individual interacting with population ("leaky Quarantine and Isolation") & $0-10 \%$ \\
\hline
\end{tabular}


Description of the social network used to simulate SAR-CoV-2 transmission at the USAF Academy:

Nodes:

- 4000 students.

- 500 faculty.

- 500 staff.

- $\quad$ Edges between nodes:

- $\quad$ Students in a student squadron.

- $\quad$ Students between student squadrons (squadron-to-squadron link).

- Faculty-to-faculty.

- Faculty-to-cadets in class.

- $\quad$ Students-to-students in class.

- $\quad$ Staff-to-staff.

- Staff-to-students.

The USAF Academy is organized around 40 student squadrons consisting of approximately 100 students each. Students live and interact within squadrons. Each student attends on average five classes. A Erdős-Rényi random (binomial) graph was used for each of the base sub-groups to connect nodes with the probability of average close contact per category. A pseudo-random graph algorithm is used to connect nodes between sub-groups with the probability of average expected close contact per category in Table A2. The result is a social network structure that closely resembles expected close contacts typical of the US Air Force Academy.

Table A2. US Air Force Academy Social Network Generation.

\begin{tabular}{ccc}
\hline Edge Relationship & Description & Value \\
\hline Student & Average close contacts per student in student squadron & 8 \\
Student & Average close contact links between student squadrons & 5 \\
Faculty & Average close contacts between faculty members & 1.5 \\
Faculty/Student & Average close contacts due to attending class (between faculty and students) & 3 \\
Student & Attends 5 classes & 5 \\
Faculty & Teaches 2 classes & 2 \\
Student & Average close contacts per class (student-to-student) & 1.5 \\
Staff & Average close contacts between staff members & 1.5 \\
Staff/Student & Average close other contacts (student-to-staff) & 1.5 \\
\hline
\end{tabular}

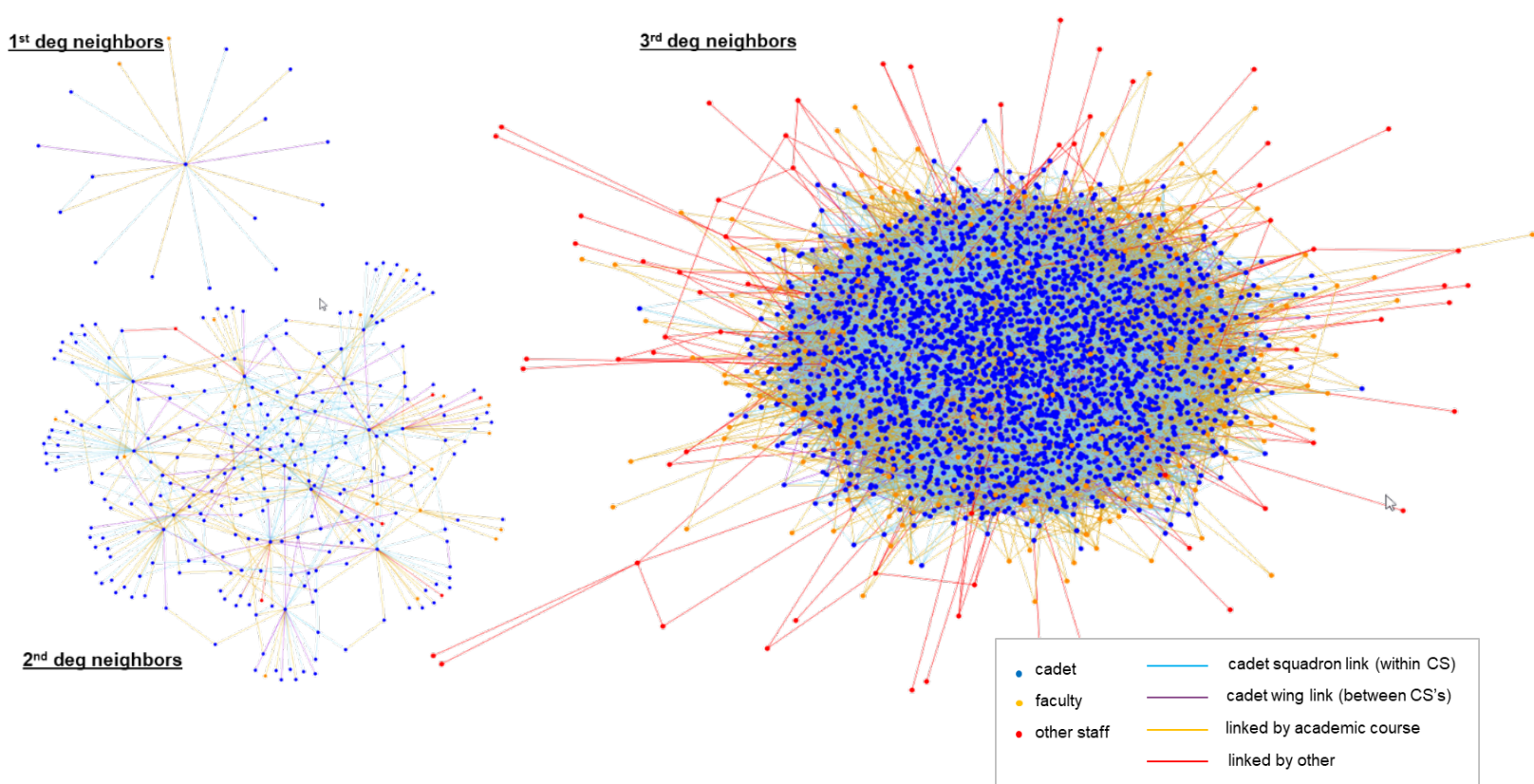

Figure A1. First-, second-, and third-degree neighbors for a random student node. 
Appendix B. Stochastic SEIR Simulation of USS Theodore Roosevelt Outbreak

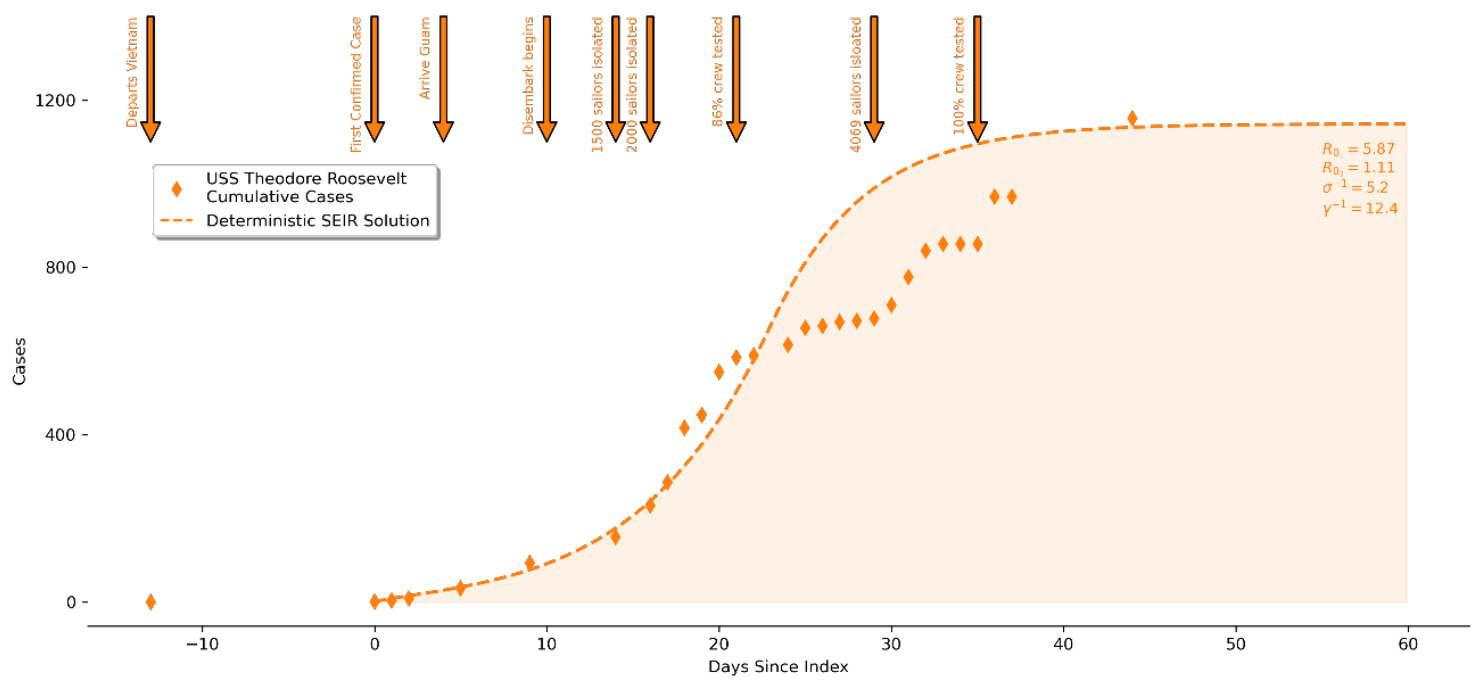

(a)

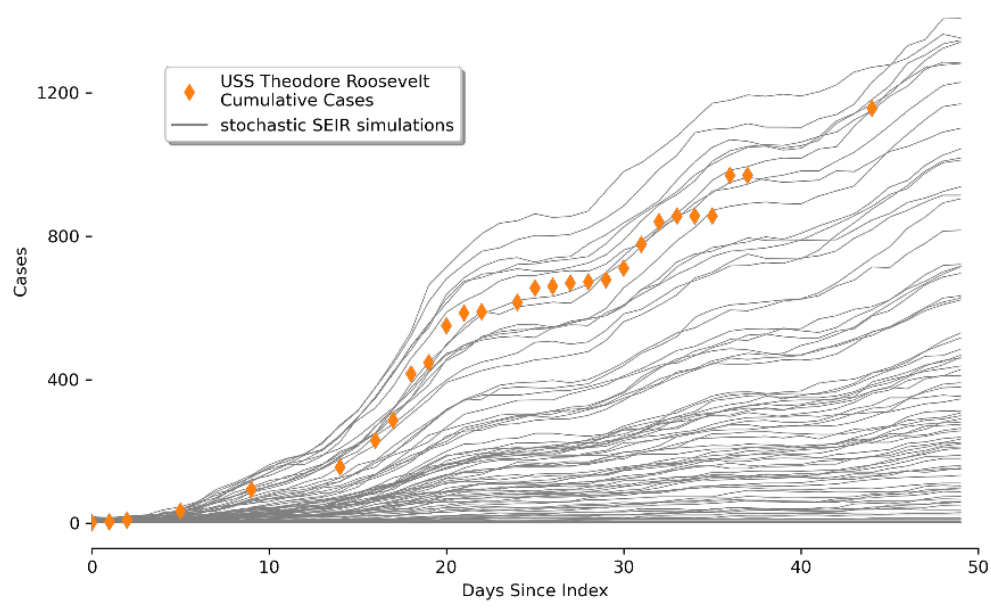

(b)

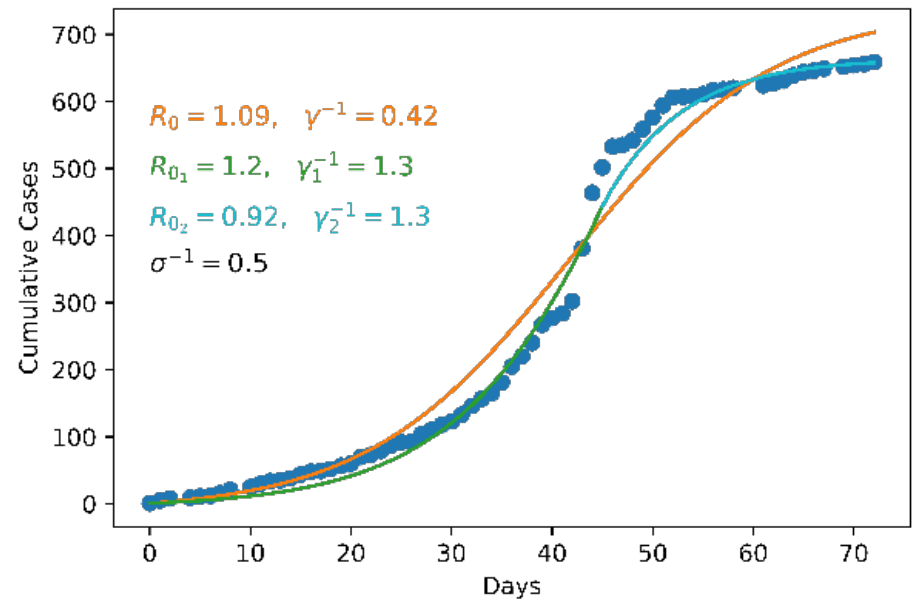

(c)

Figure A2. (a) Spring 2020 coronavirus outbreak on the U.S.S. Theodore Roosevelt Aircraft Carrier with deterministic SEIR least-squares parameter fits; (b) comparison of the stochastic SEIR computer simulations with actual outbreak data on the U.S.S. Theodore Roosevelt; (c) deterministic SEIR least-squares parameter fits to USAFA Norovirus 2019 outbreak (single fit (orange) and two piecewise fits (green, cyan)) demonstrating change in public health measure on day 42. 


\section{References}

1. Delamater, P.L.; Street, E.J.; Leslie, T.F.; Yang, Y.T.; Jacobsen, K.H. Complexity of the basic reproduction number (R0). Emerg. Infect. Dis. 2019, 25, 1-4. [CrossRef] [PubMed]

2. Barril, C.; Calsina, À.; Ripoll, J. A practical approach to R0 in continuous-time ecological models. Math. Meth. Appl. Sci. 2018, 41, 8432-8445. [CrossRef]

3. Breda, D.; Florian, F.; Ripoll, J.; Vermiglio, R. Efficient numerical computation of the basic reproduction number for structured populations. J. Comput. Appl. Math. 2021, 384, 113-165. [CrossRef] [PubMed]

4. Fauci, A.S.; Lane, H.C.; Redfield, R.R. Covid-19-Navigating the uncharted. N. Engl. J. Med. 2020, 382, 1268-1269. [CrossRef] [PubMed]

5. Mizumoto, K.; Kagaya, K.; Zarebski, A.; Chowell, G. Estimating the asymptomatic proportion of coronavirus disease 2019 (COVID-19) cases on board the Diamond Princess cruise ship, Yokohama, Japan, 2020. Eurosurveillance 2020, 25, 2000180. [CrossRef] [PubMed]

6. $\quad$ Kimball, A.; Hatfield, K.M.; Arons, M.; James, A.; Taylor, J.; Spicer, K.; Bardossy, A.C.; Oakley, L.P.; Tanwar, S.; Chisty, Z.; et al. Asymptomatic and Presymptomatic SARS-CoV-2 Infections in Residents of a Long-Term Care Skilled Nursing Facility-King County, Washington, March 2020. MMWR 2020, 69, 377-381. [CrossRef] [PubMed]

7. Nishiura, H.; Chowell, G. The Effective Reproduction Number as a Prelude to Statistical Estimation of Time-Dependent Epidemic Trends. In Mathematical and Statistical Estimation Approaches in Epidemiology; Springer: Dordrecht, The Netherlands, 2009; pp. 103-121, ISBN 9789048123124.

8. Lavezzo, E.; Franchin, E.; Ciavarella, C.; Cuomo-Dannenburg, G.; Barzon, L.; Del Vecchio, C.; Rossi, L.; Manganelli, R.; Loregian, A.; Navarin, N.; et al. Suppression of a SARS-CoV-2 outbreak in the Italian municipality of Vo'. Nature 2020, 584, 425. [CrossRef] [PubMed]

9. Bai, Y.; Yao, L.; Wei, T.; Tian, F.; Jin, D.Y.; Chen, L.; Wang, M. Presumed Asymptomatic Carrier Transmission of COVID-19. JAMA 2020, 323, 1406-1407. [CrossRef] [PubMed]

10. GitHub—Ryansmcgee/Seirsplus: Models of SEIRS Epidemic Dynamics with Extensions, Including Network-Structured Populations, Testing, Contact Tracing, and Social Distancing. Available online: https://github.com/ryansmcgee/seirsplus (accessed on 23 October 2020).

11. Payne, D.C.; Smith-Jeffcoat, S.E.; Nowak, G.; Chukwuma, U.; Geibe, J.R.; Hawkins, R.J.; Johnson, J.A.; Thornburg, N.J.; Schiffer, J.; Weiner, Z.; et al. SARS-CoV-2 Infections and Serologic Responses from a Sample of U.S. Navy Service Members-USS Theodore Roosevelt, April 2020. MMWR 2020, 69, 714-721. [CrossRef] [PubMed]

12. COVID-19 Hospitalizations. Available online: https://gis.cdc.gov/grasp/covidnet/covid19_3.html (accessed on 23 October 2020).

13. Hundreds Test Positive to COVID-19 on French Aircraft Carrier. Available online: https://www.smh.com.au/world/europe/ hundreds-test-positive-to-covid-19-on-french-aircraft-carrier-20200416-p54ken.html (accessed on 22 April 2020).

14. Van Den Driessche, P.; Watmough, J. Reproduction numbers and sub-threshold endemic equilibria for compartmental models of disease transmission. Math. Biosci. 2002, 180, 29-48. [CrossRef]

15. Ferretti, L.; Wymant, C.; Kendall, M.; Zhao, L.; Nurtay, A.; Abeler-Dörner, L.; Parker, M.; Bonsall, D.; Fraser, C. Quantifying SARS-CoV-2 transmission suggests epidemic control with digital contact tracing. Science 2020, 368. [CrossRef] [PubMed]

16. Nishiura, H.; Kobayashi, T.; Miyama, T.; Suzuki, A.; Jung, S.; Hayashi, K.; Kinoshita, R.; Yang, Y.; Yuan, B.; Akhmetzhanov, A.R.; et al. Estimation of the asymptomatic ratio of novel coronavirus infections (COVID-19). medRxiv 2020, 94, 154. [CrossRef] [PubMed]

17. Vogels, C.B.F.; Brito, A.F.; Wyllie, A.L.; Fauver, J.R.; Ott, I.M.; Kalinich, C.C.; Petrone, M.E.; Casanovas-Massana, A.; Catherine Muenker, M.; Moore, A.J.; et al. Analytical sensitivity and efficiency comparisons of SARS-CoV-2 RT-qPCR primer-probe sets. Nat. Microbiol. 2020, 5, 1299-1305. [CrossRef] [PubMed]

18. Byrne, A.W.; McEvoy, D.; Collins, A.B.; Hunt, K.; Casey, M.; Barber, A.; Butler, F.; Griffin, J.; Lane, E.A.; McAloon, C.; et al. Inferred duration of infectious period of SARS-CoV-2: Rapid scoping review and analysis of available evidence for asymptomatic and symptomatic COVID-19 cases. BMJ Open 2020, 10, e039856. [CrossRef] [PubMed]

19. Lai, C.C.; Shih, T.P.; Ko, W.C.; Tang, H.J.; Hsueh, P.R. Severe acute respiratory syndrome coronavirus 2 (SARS-CoV-2) and coronavirus disease-2019 (COVID-19): The epidemic and the challenges. Int. J. Antimicrob. Agents 2020, 55, 105924. [CrossRef] [PubMed]

20. Oran, D.P.; Topol, E.J. Prevalence of Asymptomatic SARS-CoV-2 Infection: A Narrative Review. Ann. Intern. Med. 2020, 173, 362-367. [CrossRef] [PubMed]

21. Tillett, R.L.; Sevinsky, J.R.; Hartley, P.D.; Kerwin, H.; Crawford, N.; Gorzalski, A.; Laverdure, C.; Verma, S.C.; Rossetto, C.C.; Jackson, D.; et al. Genomic evidence for reinfection with SARS-CoV-2: A case study. Lancet Infect. Dis. 2020, 21, 52-58. [CrossRef]

22. Overbaugh, J. Understanding protection from SARS-CoV-2 by studying reinfection. Nat. Med. 2020, 26, 1680-1681. [CrossRef] [PubMed] 